\title{
Solving optimization problems when designing power supply circuits
}

\author{
O. V. Shemelova ${ }^{1, *}$, E. V. Yakovleva ${ }^{1}, T$. G. Makuseva ${ }^{1}$, I. I. Eremina ${ }^{2}$, and O. N. Makusev ${ }^{1}$ \\ ${ }^{1}$ Nizhnekamsk Chemical Technological Institute (branch of Kazan National Research Technological University), Nizhnekamsk, Russia \\ ${ }^{2}$ Naberezhnye Chelny Institute of Kazan Federal University, Naberezhnye Chelny, Russia
}

\begin{abstract}
One of the quickly developing trends in the optimization of electric power grids is system development of operation and optimization of branch circuits which are based on linear programming problems. One of its categories is traffic problem. The paper discusses the formulation of various types of transport optimization problems used in the design of the most efficient power supply systems in the real sector of economy. The construction of arithmetic models of problems is carried out. Their optimality criterion is cost minimization for the design of electrical network diagrams consisting of power lines connecting sources and consumers. Examples of designing optimization power layout in mathematical problems considering the transmission capacity of power lines is given. The paper also touches upon a mathematical problem considering possible transit of capacities. The task is to build a mathematical model and solve problems that ensure minimization of process losses and losses of power when designing electrical networks. The results of solving problems are presented in the form of power supply circuits corresponding to the most optimal linking of source and consumer nodes. The work is of a scientific and practical significance as it considers the problem of optimizing economic costs when designing electric power network schemes. Moreover it is based on a qualitatively different level of use of the traffic problem algorithm. The algorithm for solving the minimization problem obtained in this paper allows developing the necessary computing operations as well as quickly obtaining the results of solving the cost optimization problem in the designed electric power network.
\end{abstract}

\section{Introduction}

Various kinds of decision-making problems lead to a variety of optimization tasks. The goal of solving optimization problems is to find the most suitable option. One of the tasks directly related to finding the optimal solution is the transportation problem.

The transportation task is the task of finding the ways of transporting the product from the points of production to the points of consumption with the minimum transportation cost [1-5].

The general mathematical terminology of the transport problem can also be applied to problems in the field of energy. In this case, the product refers to the electrical power supplied from the power source to various consumers via power transmission lines (PTL). The power source can be power plants or electrical substations, the consumers can be industrial, city-wide, agricultural and industrial consumers of electricity. In such tasks, we optimize the costs of the power supply scheme consisting of power transmission lines connecting the energy source nodes with the energy consumer nodes.

This paper discusses three types of transport optimization problems used in the design of power supply systems [1], [6-10]: 1) classical formulation of the problem; 2) transport problem with power transit; 3) transport problem taking into account transmission capacity. Let us consider each of the above tasks in detail.

\section{Setting and solving problems}

The classical formulation of the transportation problem, optimizing the cost of the electrical circuit, is as follows.

For example, in the designed electric power supply system there is $j=1,2, \ldots, m$ consumer nodes and $i=1,2, \ldots, n$ power supply nodes. The energy capacity of each of the energy sources is $A_{i}$ power units (p.u.), and the capacity of each of the energy consumers is $B_{j}$ p.u. The two-sided configuration of the nodes of energy sources and energy consumers is given initially. The unit cost of transmitting a unit of power from node $i$ to node $j$ is $z_{i j}$ conventional unit/ power units (c.u./p.u.). It is necessary to determine the optimal power supply circuit, which ensures minimum costs when using the electrical grid.

Creating a mathematical model and finding the most optimal solution to problems of this type is similar to the

\footnotetext{
* Corresponding author: olga-shemelova@yandex.ru
} 
well-known formulations of the classical problem of transportation. It is necessary to minimize the objective function

$$
z=\sum_{i=1}^{n} \sum_{j=1}^{m} z_{i j} x_{i j}
$$

where $x_{i j}$ is the required values of the transmitted power from source $A_{i}$ to consumer $B_{j}$.

At the same time, for each $i$-th power source, the sum of powers flowing through power transmission lines to all $j=1,2, \ldots, m$ consumer nodes must be equal to the power $A_{i}$ of this source.

$$
\sum_{j=1}^{m} x_{i j}=A_{i} \quad i=1,2, \ldots, n
$$

At the same time, for each $j$-th consumer, the sum of the capacities flowing along the power lines from all $i=1,2, \ldots, n$ power sources must be equal to the power $B_{j}$ of the given consumer node

$$
\sum_{i=1}^{n} x_{i j}=B_{j}, \quad j=1,2, \ldots, m .
$$

The last two equalities take into account the performance of the power balance for each node. In addition, they are also limitations of the linear model of the transportation problem. The number of restrictions should be equal to the number of nodes of sources and consumers $n+m$.

The search for the optimal solution of the problem can be accomplished, for example, using the potential method [1, 11] or with the help of the MS Excel spreadsheet processor.

In comparison with transport problems in the classical formulation, transport problems with power transit (intermediate traffic) are more general tasks and have unlimited potential possibilities for increasing the efficiency of the power supply circuit.

In the actually implemented schemes of electrical networks, it is often the most advantageous to transfer power through intermediate (so-called transit) nodes. Such transit nodes can be both a power supply node and a consumer node.

In contrast to the classical formulation of the transportation problem, transit power is indicated by a variable with two identical indices. This double number corresponds to the node number through which the power flows [1].

Characteristic features of the transportation problem with power transit are as follows:

1. all $n$ nodes of sources and $m$ nodes of consumer are numbered $1,2, \ldots,(n+m)$;

2. as a rule, intermediate (transit) power $x_{i j}$ can be transmitted through any $i$-th node;

3. unit cost of transit power transmission is $z_{i i}=0$;
4. transport matrix has the shape of a square $(n+m) \times(n+m)$;

5. transit variables $x_{i i}$ enter the solution of the problem with a negative sign;

6. regardless of the values, all transit variables are basic.

Task formulation with power transit: From the substation of an industrial enterprise located at node 1 with a capacity of $S_{1}=18$ p.u., electricity will be supplied to the workshops located at nodes 2, 3 and 4 with capacities of $S_{2}=6, S_{3}=5, S_{4}=7$ p.u. respectively. The specific costs for the transfer of power over the power transmission lines between the nodes $z_{i j}$ c.u./p.u. are also set $\quad\left(z_{12}=z_{21}=1,0\right.$; $z_{13}=z_{31}=1,0 ; \quad z_{14}=z_{41}=2,1 ; \quad z_{23}=z_{32}=1,4 ;$ $\left.z_{24}=z_{42}=1,0 ; z_{34}=z_{43}=2,3\right)$. It is necessary to determine the optimal power supply circuit. The minimum cost of the electrical network will be the optimality criterion.

In accordance with the formulation of the problem, a mathematical model was compiled that takes into account the balanced initial transport model of the problem and the rules that take into account possible power transits. The resulting mathematical model contains the objective function and the system of constraints in the form of the following equalities and inequalities.

It is required to minimize the function

$$
\begin{aligned}
Z & =1,0 x_{12}+1,0 x_{13}+2,1 x_{14}+1,0 x_{21}+1,4 x_{23}+1,0 x_{24}+ \\
& +1,0 x_{31}+1,4 x_{32}+2,3 x_{34}+2,1 x_{41}+1,0 x_{42}+2,3 x_{43}
\end{aligned}
$$

with the imposed restrictions

$$
\left\{\begin{array}{c}
x_{12}+x_{13}+x_{14}-x_{11}=18 \\
x_{21}+x_{23}+x_{24}-x_{22}=0 \\
x_{31}+x_{32}+x_{34}-x_{33}=0 \\
x_{41}+x_{42}+x_{43}-x_{44}=0 \\
x_{21}+x_{31}+x_{41}-x_{11}=0 \\
x_{12}+x_{32}+x_{42}-x_{22}=6 \\
x_{13}+x_{23}+x_{43}-x_{33}=5 \\
x_{14}+x_{24}+x_{34}-x_{44}=7 \\
x_{i j} \geq 0 \quad \forall \quad i, j
\end{array}\right.
$$

To solve the problem, a tabular processor MS Excel was also used. With the help of the add-on "Search for a solution" an optimal solution was found.

Figure 1 shows the relative position of the source node $S_{1}$ and consumer nodes $S_{i}(i=2,3,4)$. When the optimal solution with the possible transit node was found, an optimal solution was obtained and a power supply network diagram was constructed. 


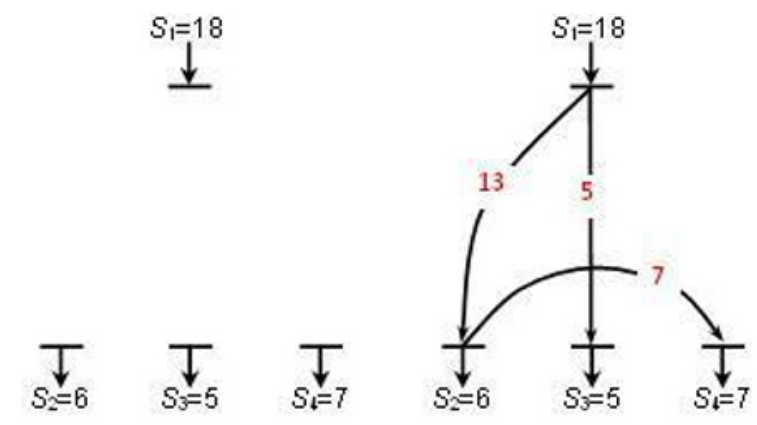

Fig. 1. Electric power supply (EPS) circuit in the problem with power transit.

In electrical engineering and power engineering in general there also arises the problem of taking into account the transmission capacity of power transmission lines. Hence it is very important to take into account the limitations of the capacity of the lines at the stage of designing the electric power supply circuit. In particular, defining the power transmitted over an existing line is determined by the amount of permissible heating of the wires of power lines [1].

The formulation of a task taking into account the transmission capacity: Source power $a_{1}=40$, $a_{2}=10, a_{3}=30, a_{4}=20$ p.u. and loads $b_{1}=50$, $b_{2}=10, b_{3}=30, b_{4}=10$ p.u., as well as unit costs $c_{i j}$ c.u./p.u. $(i, j=1,2,3,4)$ to transfer electricity from each source $i$ to each consumer $j\left(c_{11}=6, c_{12}=3\right.$, $c_{13}=4, c_{14}=5, c_{21}=5, c_{22}=2, c_{23}=3, c_{24}=3$, $c_{31}=3, c_{32}=4, c_{33}=2, c_{34}=4, c_{41}=5, c_{42}=6$, $c_{43}=2, c_{44}=7$ ) are known for the power system. At the same time, for line $x_{43}$ between source $A_{4}$ and consumer $B_{3}$, the transmitted power is limited to $S=7$ p.u. $\left(x_{43} \leq 7\right)$. It is necessary to plan the electrical network with the lowest cost (it is possible to lay air lines only from the source to the loads) [12].

The formulation of this problem requires the compilation of a mathematical model that takes into account a balanced transport model of the problem. The constructed model contains the objective linear function and the system of constraints in the form of equalities and inequalities.

The solution was found directly for the task without limitations and for the task taking into account the capacity of the power transmission lines. To find solutions, a tabular processor MSExcel was used.

Figure 2 shows the relative position of nodes of sources $A_{i}(i=1,2,3,4)$ and consumers $B_{j}(j=1,2,3,4)$. In the classical formulation of transport problems, the optimal electric power supply circuit looks like that shown in Fig. 2. When searching for the optimal solution with regard to the transmission capacity, a solution was obtained and the electric power supply circuit shown in Figure 3 was constructed.

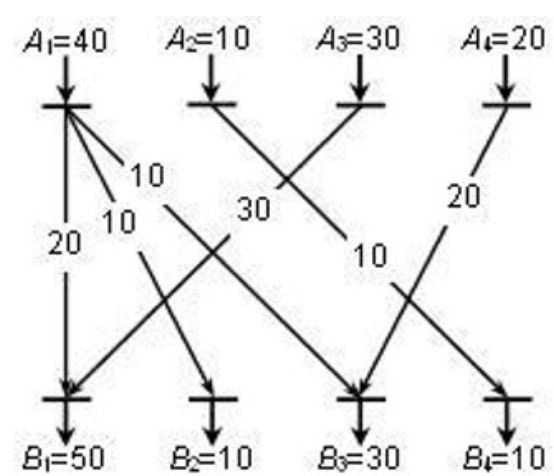

Fig. 2. EPS circuit without taking into account the transmission capacity (total network costs 350 c.u./p.u.).

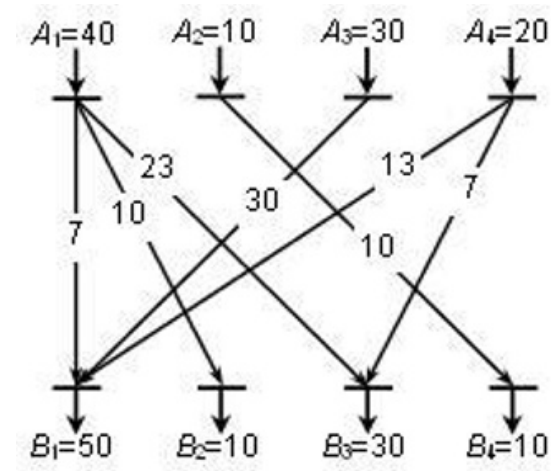

Fig. 3. EPS circuit in the problem taking into account the transmission capacity (total network costs 350 c.u./p.u.).

Let us consider the task of developing a costeffective plan for the distribution of electricity from electricity generating stations to certain localities.

Task statement: Three generating stations producing electricity $C_{1}=25 \mathrm{GW} \cdot \mathrm{h}, \quad C_{2}=40 \mathrm{GW} \cdot \mathrm{h} \quad$ and $C_{3}=30 \mathrm{GW} \cdot \mathrm{h}$ supply three settlements with electricity. It is known that the maximum electricity needs of these settlements are estimated at $P_{1}=30 \mathrm{GW} \cdot \mathrm{h}, P_{2}=35$ $\mathrm{GW} \cdot \mathrm{h}$ and $P_{3}=25 \mathrm{GW} \cdot \mathrm{h}$. It is also known that the costs of transmitting power over power transmission lines between stations and settlements are $z_{i j}$ c.u. $/ \mathrm{GW} \cdot \mathrm{h}$ $\left(z_{11}=600, \quad z_{12}=700, \quad z_{13}=400, \quad z_{21}=320\right.$, $z_{22}=300, \quad z_{23}=350, \quad z_{31}=500, \quad z_{32}=480$, $\left.z_{3}=450\right)$. At certain times of the year, the need for electricity in each of these three settlements increases by $20 \%$. In this case, it is assumed that the lack of electricity can be replenished from another power supply network at a higher cost (1000 c.u./GW·h). However, the third settlement cannot join another power grid. Power plants are planning to design a more cost-effective program that provides electrical balance between consumers. It is necessary to plan the lowest-cost electrical network (it is possible to lay air lines only from the source to the loads) and determine the cost of additional electricity for each of these settlements at a certain time of the year. 
To solve the problem, the corresponding mathematical models were compiled, which take into account the unbalanced initial transport model of the problem. [6].

The solution was found for the initial formulation of the problem, as well as for the problem, taking into account the settlements' increased need for additional power capacity. To find solutions, a tabular processor MSExcel was used.

Figure 4 shows the mutual arrangement of the nodes of the $C_{i}(i=1,2,3)$ electricity generating stations and consumers: the settlements of $P_{j}(j=1,2,3)$. In the initial formulation of the problem, the optimal electric power supply circuit has the form shown in Fig. 4 . When searching for the optimal electricity distribution plan, taking into account the increase in demand at a certain time of the year, a solution was obtained and the following electric power supply circuit was constructed (Figure 5).

At the same time, the cost of additional energy for each of these settlements at a certain time of the year will be the following:

the first settlement will spend $500 \cdot 23+1000 \cdot 13=24500$ c.u. (it is at 10400 c.u. more than in other months);

second settlement will spend: $300 \cdot 40+480 \cdot 2=12960$ c.u. (it is at 2460 c.u. more than in other months);

the third settlement will spend: $400 \cdot 25+450 \cdot 5=12250$ c.u. (it is at 2250 c.u. more than in other months).

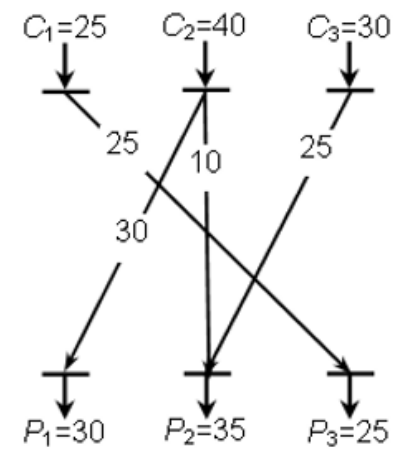

Fig. 4. EPS circuit in the task of providing electricity to settlements (total network costs 34600 c.u./GW·h).

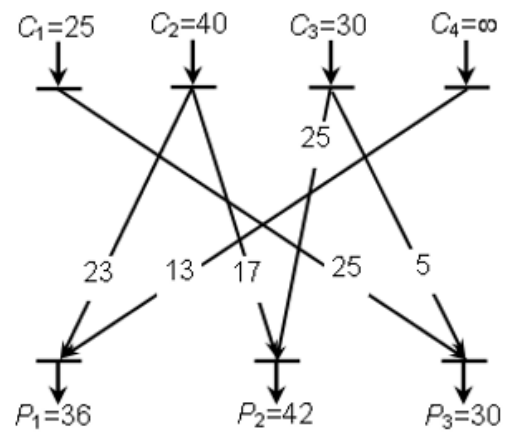

Fig. 5. EPS circuit in the task, taking into account the provision of additional electricity to settlements (total network costs 49710 c.u./GW·h).

\section{Conclusions}

Thus, transport tasks in the field of energy are used in the design of power and electricity supply schemes. At the same time, the tasks are set to optimize the topology, structure, operating modes and the use of electrical networks as well as to reduce technological losses and power losses with modern technologies. Therefore, it can be noted that the electric power industry has many alternative ways of development, and the solution of the transportation problem is an essential part of the optimization of activity in the electric power industry [1, $11]$.

It should be noted that the ability to solve optimization tasks can act, on the one hand, as a kind of «production discovery» that ensures minimal costs when using electrical networks, and on the other hand, is a means of forming «so-called engineering competence, by which we mean integrative professional personality characteristics. From this point of view, the improvement of the system of engineering and technical training means not only the possession of modern technologies, knowledge of modern equipment, etc., but the possession of certain personal qualities» [13].

It should be emphasized once again that the most effective way to comprehensively form engineering competence is to build a competency model for a discipline that «allows you to manage the learning process: out of bricks, students are «built» into qualified graduates who are capable of continuous selfdevelopment, cultural, entrepreneurial, initiative, independent in thinking and acting, creating the most valuable resource of the state - human capital» [14].

Of course, within the framework of this article we did not try to cover all possible optimization methods when designing power supply systems, we only showed a variety of approaches based on the understanding of the solution of the transportation problem in the field of energy. In the future, when planning power supply systems, we consider it expedient to proceed from solving transport optimization problems. In this regard, «there is a need to modernize the methods and tools accompanying the learning process, allowing students to become familiar with the main trends of informatization of the professional sphere, to master the theoretical foundations of the discipline, hardware and software, practical skills of using information technology in their professional activities", - in its turn, it will allow improving the quality of solving problems of constructing power supply system circuits [15].

\section{References}

[1] V.N. Kostin, Optimization problems of electric power industry (St. Petersburg: SZTU) (2003)

[2] E.I. Gracheva, O V.Fedorov, Forecasting reliability electrotechnical complexes of in-plant electric power supply taking into account low-voltage electrical apparatuses 2019 International Conference on Industrial Engineering, Applications and Manufacturing, ICIEAM 2019, 8743057 (2019) 
[3] A. M. Kopylov, I.V. Ivshin, A.R. Safin, R.S. Misbakhov, R.R. Gibadullin, Assessment, calculation and choice of design data for reversible reciprocating electric machine, International Journal of Applied Engineering Research, 10(12), 31449-31462 (2015)

[4] I.G. Akhmetova, N.D. Chichirova, Mathematical modeling of the process of determining the standards for process losses in the transfer of thermal energy of the coolant, Journal of Physics: Conference Series, 891(1),012168 (2017)

[5] E.I. Gracheva, O.V. Naumov, Probabilistic forecasting changes in magnitude of insulation resistance of line cable and wire workshop networks, Proceedings 2018 International Conference on Industrial Engineering, Applications and Manufacturing, ICIEAM 2018, 8728840 (2018)

[6] O.M. Grunin, Savitskiy, Mathematical problems of energy (Chita: ZabGU), 2014

[7] E.I. Gracheva, O.V. Fedorov, Forecasting reliability electrotechnical complexes of in-plant electric power supply taking into account low-voltage electrical apparatuses, 2019 International Conference on Industrial Engineering, Applications and Manufacturing, ICIEAM 2019, 8743057 (2019)

[8] I. Sultanguzin, A. Fedyukhin, V. Karasevich, A. Chernykh, Implementation of compressed air energy storages (CAES) for increasing efficiency of natural gas usage for power generation, International Gas Research Conference Proceedings, 2, 1193-1198 (2017)

[9] E.I. Gracheva, O.V. Naumov, Researches of indicators reliability and probabilistic characteristics of elements electric equipment of shop electric networks, 2016 2nd International Conference on Industrial Engineering, Applications and Manufacturing, ICIEAM 2016 - Proceedings, 7910975 (2016)

[10] Y.N. Zvonareva, Y.V. Vankov, Energy saving in systems of heat supply of the large municipal associations powered from several sources of heat, Bulletin of the Tomsk Polytechnic University, Geo Assets Engineering, 326(11), 75-82 (2015)

[11] O.M. Grunin, Savitskiy Mathematical problems of energy (Chita: ZabGU) (2014)

[12] V.N. Laptev, V.V. Stepanov, V.A. Atroschenko, Y.A. Kabankov, M.V.Stepanova, To the questions of finding the optimal power loss electric networks based upon solutions of transport problems by the potential method Scientific Journal of KubSAU 120: 06 1-18 (2016)

[13] O.V. Shemelova, The simulation of the solution of optimal plans constructing in electric power supply systems Handbook. An Engineering Journal 11, 260 28-32 (2018)

[14] T.G. Makuseva, General cultural component as a way to form engineering competencies International Conference on Interactive Collaborative Learning, Kazan 513-514 DOI: 10.1109/ICL.2013.6644638 (2013)

[15] O.N. Makusev, L.V. Bakeeva, Implementation of the principles of uniqueness of each person and of the openness of the educational systems in higher education Bulletin of the Kazan Technological University 17: 11, 293-296 (2014)
[16] I.I. Eremina, E.V. Abrosimova, Problems of formation of competency modeling, application and information process of enterprises and organizations in preparing it professionals of the economic sector at the university Modern Journal of Language Teaching Methods 7: 10, 43-48 (2017) 